\title{
Title Model Ontology for Future Internet Networks
}

\author{
Joao Henrique de Souza Pereira ${ }^{1}$, Flavio de Oliveira Silva ${ }^{1}$, \\ Edmo Lopes Filho ${ }^{2}$, Sergio Takeo Kofuji ${ }^{1}$, and Pedro Frosi Rosa ${ }^{3}$ \\ 1 University of Sao Paulo, Brazil \\ joaohs@usp.br, flavio@pad.lsi.usp.br, kofuji@pad.lsi.usp.br \\ 2 Algar Telecom, Brazil \\ edmo@algartelecom.com.br \\ 3 Federal University of Uberlandia, Brazil \\ pedro@facom.ufu.br
}

\begin{abstract}
The currently Internet foundation is characterized on the interconnection of end-hosts exchanging information through its network interfaces usually identified by IP addresses. Notwithstanding its benefits, the TCP/IP architecture had not a bold evolution in contrast with the augmenting and real trends in networks, becoming service-aware. An Internet of active social, mobile and voracious content producers and consumers. Considering the limitations of the current Internet architecture, the envisaged scenarios and work efforts for Future Internet, this paper presents a contribution for the interaction between entities through the formalization of the Entity Title Model.
\end{abstract}

Keywords: Entity, Future Internet, Ontology, Title Model

\section{Introduction}

The Internet of today has difficulties to support the increasing demand for resources and one of the reasons is related to the restricted evolution of the TCP/IP architecture since the 80s. More specifically, the evolution of the layers 3 and 4 , as discussed in 23. The commercial usage of Internet and IP networks was a considerable obstacle to the improvements in the intermediate layers in this architecture.

The challenges to Future Internet Networks are the primary motivation to this paper and the cooperation in the evolution of computer networks, specifically in the TCP/IP intermediate layers, is another one. The purpose is to present the Entity Title Model formalization, using the OWL (Web Ontology Language), to collaborate with one integrated reference model for the Future Internet, including others projects efforts.

This paper is organized as follows: Section 1 presents works in the area of Future Internet and ontology in computer systems. Section 2 describes the concepts of the Entity Title Model and the ontology at network layers. Finally, section 3 presents some concluding remarks and suggestions for future works. 


\section{Future Internet Works}

A Future Internet full of services requirements demands networks where the necessary resources to service delivery are orchestrated and optimized efficiently. In this research area there are extensive number of works and projects for the Future Internet and some of these are being discussed in collaboration groups like FIA, FIND, FIRE, GENI and others 10, 11, 14, 31, 32.

At this moment, several research groups are working towards a Future Internet reference architecture and the Title Model ontology is a contribution to this area. Projects, among others, like 4WARD, ANA, PSIRP and SENSEI proposes new network architectures which contains collaborative relations to the model proposed by this paper [1] 3] [8] 30, 33. The 4WARD Netinf concept is related to the Domain Title Service (DTS) proposed in [26] and its horizontal addressing can leverage Netinf concept. The DTS can deal with the information and with the context of the consumers taking into account their communication needs at each context, supporting their change over time.

The Entity Title Model concepts can be used at the communications layer to the real world architecture envisaged by SENSEI 33 project, besides that, the concept of addressing by use of a Title is suitable for real world Internet and its sensor networks. The title concept can be used at the publish and subscribe view proposed by PSIRP [30] and used in conjunction with its proposed patterns providing new important inputs to the content-centric view of Future Internet.

\subsection{Some Other Future Internet and Ontology Works}

Studies and proposals for development of the intermediate layers of the TCP/IP architecture are being discussed since the $80 \mathrm{~s}$, but there is still no clear and definite perspective about which standard will be used in the evolution of this architecture.

In the area of the evolution of intermediate layers of the TCP/IP there are proposals as LISP (Locator Identifier Separation Protocol), which seek alternatives to contribute to the evolution of computer networks. In the proposed implementation of LISP there is low impact on existing infrastructure of the Internet since it can use the structure of IP and TCP, with the separation of Internet addresses into Endpoint Identifiers (EID) and Routing Locators (RLOC) 9 .

In the area of next generation Internet there is also the works of Landmark developed by Tsuchiya, that proposed hierarchical routing in large networks and Krioukov work on compact routing for the Internet. Pasquini proposes changes in the use of Landmark with RoFL (Routing on Flat Labels), and flat routing in binary identity space. He also proposes the use of domain identifiers for a next-generation Internet architecture 21] 22.

Previous studies in RoFL were presented by Caesar who also made proposals in IBR (Identity-based routing) and VRR (Virtual Ring Routing) [7]. In the area of mobility on a next-generation Internet Wong proposes solutions that include support for multi-homing [36]. In this area, there are also proposals 
by Ford, who specifies the UIP/UIA (Unmanaged Internet Protocol) and UIA (Unmanaged Internet Architecture) [12.

Related to ontology, there are extensive studies in philosophy, whose concept of this term is assigned to Aristotle, who defines it as the study of "being as being". However, the name ontology was first used only in the seventeenth century by Johannes Clauberg [2]. In the area of technology its initial use was performed by Mealy in 1967 20] and expanded especially in areas of artificial intelligence, database, information systems, software engineering and semantic web. In the technology area one of the most commonly used definitions is from Tom Gruber, who defines it as "the explicit representation of a conceptualization" [15].

In technology, the use of ontology is also associated with formalizations that allow technological systems to exchange concepts. For these formalizations there are extensive literature which defines different languages and tools. As examples of languages used there are DAML, OIL, KIF, XSLT, KM, Predicate Calculus of First Order, Propositional Logic, Ontolingua, Loom, and Semantic Web languages (RDF, RDFS, DAML+OIL, OWL SPARQL, GRDDL, RDFa, SHOE AND SKOS), among others [13.

For communication between network elements, ontology is usually used in the application layer, without extending to the middle and lower layers of computer networks. In this research area, this paper aims to contribute to advancing the use of ontology to the intermediate layers as a collaborative proposal for the Future Internet.

\section{Ontology at Network Layers}

Ontologies can use layer model or distinct architectures, however, in general, they remain restricted to the application layer. For example, the architecture of the Web Ontology Language defined by W3C, presented in Fig. 1 extracted from [17, is confined in the application layer of the TCP/IP architecture.

\begin{tabular}{|c|c|}
\hline \multicolumn{2}{|c|}{ Applications } \\
\hline $\begin{array}{c}\text { Ontology Languages (OWL Full, } \\
\text { OWL DL and OWL Lite) }\end{array}$ \\
\hline RDF Schema & Individuals \\
\hline RDF and RDF/XML \\
\hline XML and XMLS Datatypes \\
\hline URIs and Namespaces \\
\hline
\end{tabular}

Fig. 1. Architecture of Web Ontology Language [17. 
In the use of TCP/IP, there are limitations concerning the application layer informing its needs to the transport layer. This occurs because in the TCP/IP architecture there are rules defined in the specification of the transport and network layers protocols to establish communication among the network elements. For example, the applications can select the protocol UDP or TCP, according to delivery guarantee, but they cannot tell the transport layer its needs of encryption or mobility.

It is possible to change the paradigm of client-server communication and the structure of the intermediate layers of the TCP/IP, so that the communication networks have expansion possibilities to support the needs of the upper layer. For so, one solution is to use an intermediate layer conceptually capable of communicating semantically with the top layer and translating these needs in the communication with and between the lower layers. A possibility proposed by the Entity Title Model.

\subsection{Entity Title Model Concepts and Semantics}

The use of ontology for model formalization needs clear definitions of the used concepts to build properly the ontology of the approached model. Thus, for the Entity Title Model its main terms concepts are:

Entity: Element whose communication needs can be semantically understood and supported by the service layer and subsequent lower Link and Physical layers. Examples of real world entities in the title model are: application, content, host, user, cloud computing and sensor networks. The notion of entity in the Title Model differs from the notion of resources in some relevant literature, as the entity here is a communication element and not one resource in a network. In this concept, the entities in the Title Model are not obligated to provide resources and can consume them. For example, one user, that demands resources, is one communication entity in the Title Model. Also, applications that do not offer resources, but demand some ones, are entities.

However, for an ontology there is correlation of the terms "Entity" and "Thing", as described in [13], where "Entity" or "Thing" in an ontology refers to its first class, which is the superclass of all other classes.

For the taxonomy of the ontology, the classification of an entity in the Entity Title Model can expand the categories as application, content, cloud, sensor, host, user. Also can be created other kinds of classification, such as hardware, software and network, among others. Some one of them (not all) can be used as resources in others relevant literature.

As the root superclass of one ontology is "Entity" or "Thing" the Entity Title Model ontology designates a conceptually different "Entity" of this model, which in turn is an communication element that have its communication needs understood and supported by computer networks. For example, in this taxonomy the class "layer" is a subclass of "Thing" and neither this class nor its subclasses are entities to the Entity Title Model, although the class "Layer" is an entity to the concept of ontology, in general. 
Title: It is the only designation to ensure an unambiguous identification. An unique identity. The Entity Title Model proposes that the use of titles of applications, specified in the ISO-9545/X.207 recommendation, be extended to the other communication entities of the computer networks. According to this recommendation, the ASO-title (Application Service Object-title), which are used to identify with no ambiguity the ASO in an OSI environment, consists of AP-title (Application Process title) which, by nature, addresses the applications horizontally [16].

This work broadens the use of the title from the applications with the unification of addresses by using the AP-title and also suggests that the intermediate layers support the needs of the entities in a better way, with the purpose of improving the addressing of internet architecture by horizontal addressing and facilitate communication among the entities and with the other layers 24]. Not to use a separate classification for "user title", "host title" and "application title", which would reduce the flexibility of its use in other addressing needs (eg, grid title, cluster title and sensor network title), this model defines de use of the single designation "entity title" or simply "title", whose goal is to identify an entity, regardless of which one it is.

Entity Title: It is the sole designation to ensure the unambiguous identification of a communication element whose needs may be semantically understood and supported by the service layer and subsequent lower link and physical layers. Examples of entity title are: Digital Signature, DNA, e-mail address and hash.

Layer: It designates the concept to explain the general ideas of abstraction of the complexities of a problem under its responsibility. A layer deals internally with the details under its responsibility and has an interface with the adjacent neighboring layers. The Entity Title Model layers are: Physical, Link, Service and Entity.

Entity Title Model: It is the 4-layer model that defines the entity layer as the upper layer, whose communication needs are semantically understood and supported by the service layer (intermediate layer) that has the physical and link layers as subsequent lower ones.

Link: It is the connection between two or more entities.

Physical: It is a tangible material in a computer network, such as: cables, connectors, general optical distributor, antenna, base station and air interface.

Service: It is the realization of the semantics of the need of a communication element, based on "service concept" presented by Vissers, where users communicate with each other through a "Service Provider", whose interface is accomplished by a "Service Access Point" (SAP) 34. In the Entity Title Model the "entity" is the "user" of the Vissers service model and the "Service Layer" is the "Service Provider". In the Entity Title Model the SAP is formalized with the use of ontology, which in this work was built in OWL.

Needs: They are functionality or desirable technological requirements, essential or indispensable.

Entity Needs: They are functionality or desirable technological requirements, essential or indispensable for the communication elements whose needs 
can be semantically understood and supported by the service layer and subsequent lower link and physical layers. Examples of needs of the entities are: Low latency, low jitter, bandwidth, addressing, delivery guarantee, management, mobility, QoS and security.

The changing needs of the entities may vary depending on the context of the entities in communication, and also the context of communication itself. The contexts can be influenced by space, time, specific characteristics of entities, among other forms of influence. Discussion on the changing needs are presented in [24], where associations between elements of communication may vary according to their desired needs and their variation in time.

Regardless of the time, the nature of communication can also influence the desired values for the facets. For example, to transfer data from a file, or content of email / instant message, it is necessary to have delivery guarantee in communication. On the other hand, for an audio or video communication in real time, it will not necessarily be important the delivery guarantee, as other needs will be most desirable, such as low jitter and low latency.

Horizontal Addressing: Possibility of having neighborhoods regardless of physical or logical location of entities in computer networks, without the need of reserved bandwidth, networks segmentation, specific physical connections or virtual private network.

Entity Layer: This is the layer that has the responsibility on the part of the problem corresponding to the elements of communication, whose needs can be understood and semantically supported by the service layer and subsequent lower link and physical layers.

Service Layer: This is the layer that has the responsibility to understand the needs of the entity layer and translate them into functionality in computer networks.

Link Layer: This is the layer that has the responsibility to establish the link between two or more entities and ensure that data exchange occurs at the link level and takes place according to the understanding made by the service layer.

Physical Layer: This is the layer that has the responsibility of the complexities of real-world tangible materials. For example, this layer has responsibility for: The levels of electrical, optical and electromagnetic signals, shape of connectors and attenuation.

Domain Title Service (DTS): It is a domain able to understand and record instances of entities and their properties and needs, facilitating communication services among them. This domain has world-wide coverage and hierarchical scalability formed by elements of local communication, masters and slaves, similar to DNS (Domain Name System). The DTS does the orchestration of the entities communication, as showed in Fig. 2.

\subsection{Cross Layer Ontology for Future Internet Networks}

For intermediate semantic layer, this work did the creation of an ontology for the Entity Title Model, considering others works and projects efforts for Future Internet, as 4WARD, Content-Centric, User-Centric, Service-Centric and AutoI 


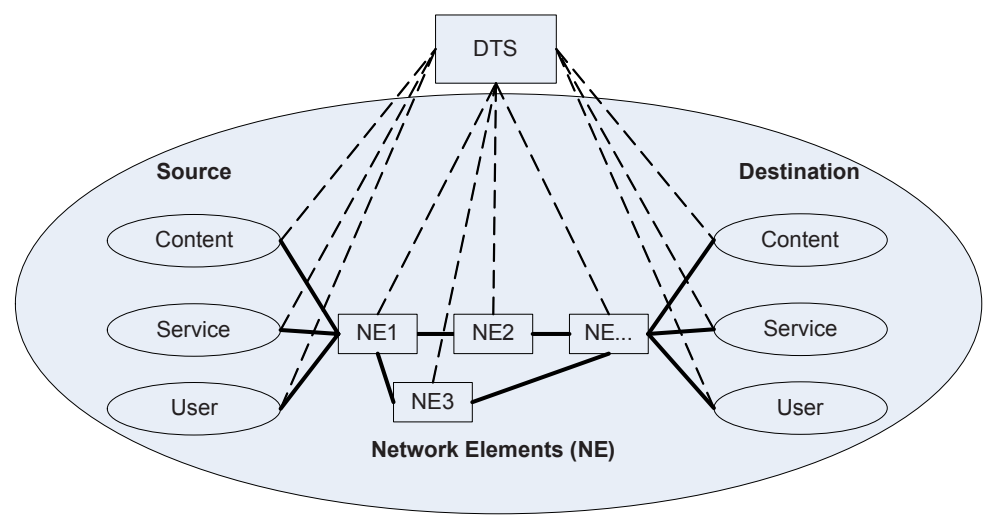

Fig. 2. Entities Communication Orchestrated by the DTS.

44] 28. This ontology also supports the proposal of Horizontal Addressing by Entity Title, presented in [26], as well as the semantic approaching cross layers for the Future Internet.

The Horizontal Addressing by Entity Title has limitations related with the communications needs formalization and standardization, and also has limitations with the collaboration with others Future Internet projects efforts. The reason is because the solution for horizontal addressing and communication needs was represented and supported using the Lesniewski Logic [18 29. The benefits for the use of the propositional logic for network formalization is the implementation facility in software and hardware. However, in a collaborative effort to others Future Internet works, the Entity Title Model has better contributions by the use of a more expressive and standardized representation language.

Also, this Model is more complete than the solution for just the horizontal addressing, as it formalize the concepts to the intermediate layers interwork and support to approaches like the Content, Service and User Centric. In addition, it permits semantic communication cross layers to contribute with, for example, the autonomic management, as the AutoI works. These are also limitations from the previous Horizontal Addressing by Entity Title works with value added by the Entity Title Model.

Others actual researches show the use of ontologies at different network layers like: OVM (Ontology for Vulnerability Management) to support security needs 35] NetQoSOnt (Network QoS Ontology) to meet the needs of service quality [27]; OOTN (Ontology for Optical Transport Networks) for use in the lower layers 6]; Ontology for management and governance of services [5. However, these studies does not use the ontology to the formalization of concepts for replacement of the intermediate layers of the TCP/IP (including its major protocols such as IP, UDP and TCP).

In the Entity Title Model, entities, regardless of their categories, are supported by a layer of services. It is very important to highlight that the name "service" in the "service layer", does not intend to conflict with the traditional 
meaning of "service concept" as, in general, the layers also expose services to other layers. In its concept, the service layer is able to understand and meet the entities needs. Fig. 3 shows the Entity Title Model layers compared with the TCP/IP and the extension of the semantic power, cross layers, enabled by the Entity Title Model.
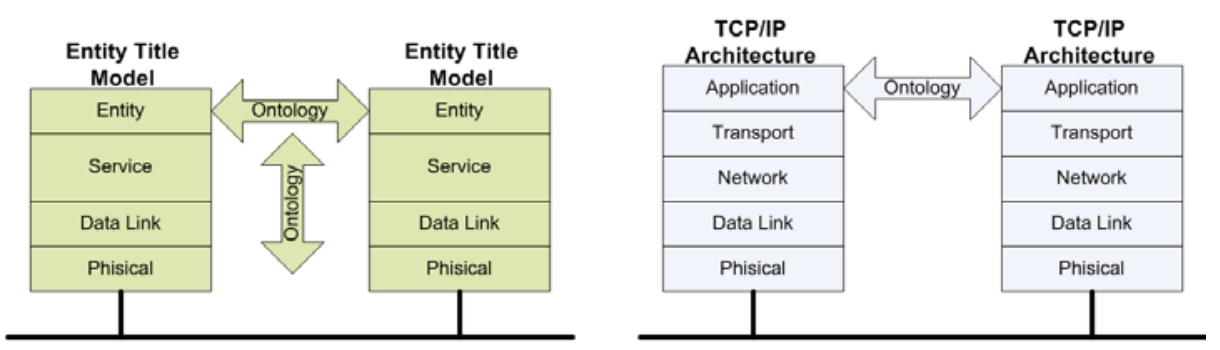

Fig. 3. Semantic Extension Cross Layers in the Title Model and the Semantic in TCP/IP.

The relationship between Entity, Services and Data Link layers are made by the use of concepts directly represented in OWL. For the communication between the layers running in a Distributed Operating System, without the traditional sockets used in TCP/IP, is used the Raw Socket to enable the communication [19.

The following OWL sample code shows one use case example for distributed programming, where the application entity with title Master-USP-1 sends its needs to the Service Layer. These needs include: Communication with SlaveUSP-A; Payload Size Control equal to 84 Bytes; and; Delivery Guarantee request. In this context, this need is informed, to the Service Layer, by the direct use of the Raw Socket to communicate with the Distributed Operating System, without the use of IP, TCP, UDP and SCTP.

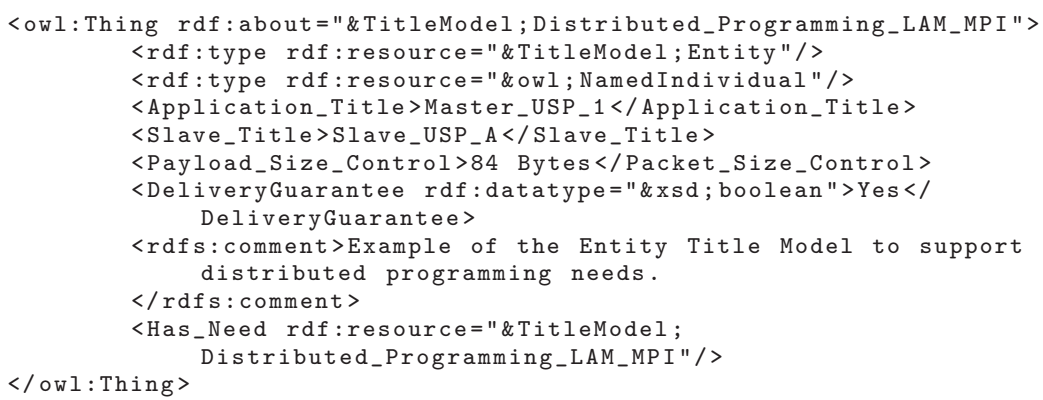

By this semantic information, the Service and Data Link layers can support the distributed programming communication using different approaches, as the addressing proposal presented in [25. However, the use of the Entity Title Model is independent from the addressing way used. For example, the works related to Generic Path, Information Channels, RoFL and LISP can use it, but some of 
them, as RoFL and LISP, should change their structure to semantically support the entities needs and identification, unified in title, and not only the addressing of hosts or applications. Others works as, for example, 4WARD, AutoI OSKMV planes (Orchestration, Service Enablers, Knowledge, Management and Virtualisation planes) and the Content-Centric can use this model collaboratively.

The context name in the Content-Centric project is expanded by the title concept in the Entity Title Model, as in this model it is possible to address contents and also others entities. This can benefits the Content-Centric works to address the content by name (or title) as, in some situations, one user may need the Content directly from Services or from other Users (thoughts). In this perspective, the Entity Title Model and its ontology can contribute to converge some Future Internet projects, as the Content, Service and User Centric works, monitored and managed by the OSKMV planes using semantics cross layers, and not only in the application layer as happen in the TCP/IP architecture.

In this example for the contribution with the Content, Service and User Centric works, in the Title Model it is possible the unification of the different entities address in the Future Internet. This means that application, content, host and user can have its needs supported and can be located by its title.

By this possibilities, this work aims to contribute with the discussions for a collaborative reference model in the Future Internet, that includes different categories of communication entities, and its needs. One basic sample of the taxonomy for this "Entity" concept is showed in the Fig. 4, extracted from the Title Model ontology built in Protégé.

In this taxonomy "title" is one facet of the concept Entity and one individual of Entity has "title".

For the service layer to support semantically the entities needs this work uses the Web Ontology Language, so that the Entity layer can communicate semantically with the Service layer, which translates this communication in functionality

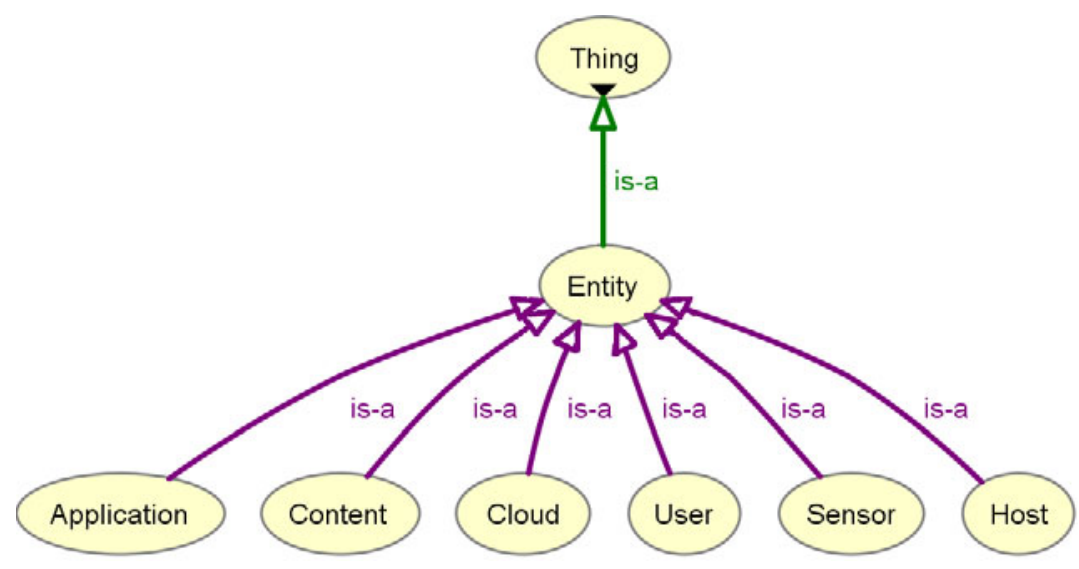

Fig. 4. Entity Taxonomy in the Title Model. 
through the Physical and Link layers. OWL was used because of its significant use in current and future trend, since its adoption and recommendation by the W3C [17.

By the Entity Title Model, some current needs of the applications are to be met in a more natural and less complex way. For example, once the title addresses the entities horizontally, the mobility on the Internet becomes natural, since there is no longer the hierarchy of segments of the network and sub network that occurs in the IP address with the use of masks. By this, the coupling between the neighborhoods are reduced, so, an entity and its neighbors can be naturally distributed anywhere in the world.

Besides reducing the complexity of the multiple addresses used in the current architecture, the use of the Entity Title Model solves the problem of the number of possible addresses, as it makes an unlimited number of addresses, since in this proposal each entity has an unique identification, through its title, without defining the amount of possible characters, or bits.

\section{Conclusion}

Studies in ontology in the technology area are used, in most part, in the application layer of TCP/IP architecture, with few studies in the lower and middle layers of this architecture. In this scenario, this work contributes to the use of ontology in the middle layers of the Internet, with the proposal of semantic formalization, in computer networks, for the Entity Title Model.

Therefore, it is possible the approaching between the upper and lower layers. As a result there is improvement in the exchange of meanings between the layers through the use of Entity and Service layers. This is a possible contribution to the Future Internet efforts and projects like AutoI, Content-Centric, User-Centric, Service-Centric, 4WARD and others. Also, is a possibility for the collaborative discussions about the reference model related to these, and others, Future Internet efforts.

As future work there will be continued the development of this ontology and its collaborative perspective with others Future Internet efforts and projects. It is suggested to extend discussions and studies concerning the unique identification of the entities and the formalization of security mechanisms for the Entity Title Model. Also, the interoperability, scalability and stability test cases for this model.

It is also suggested the continuity of studies and discussions on the use of semantic representation languages in place of protocols in the lower and middle layers of computer networks, thereby defining the communication architecture whose study go over the definitions in the area of protocols architecture.

Acknowledgment. Part of the results of this work received contributions from the MEHAR Project researches. The authors would like to thank the MEHAR Project members for all discussions and collaboration. 
Open Access. This article is distributed under the terms of the Creative Commons Attribution Noncommercial License which permits any noncommercial use, distribution, and reproduction in any medium, provided the original author(s) and source are credited.

\section{References}

[1] 4WARD: 4WARD. European Union IST 7th Framework Programme, http://www.4ward-project.eu (2011)

[2] Abbagnano, N.: Dicionário de Filosofia, Trad. Alfredo Bosi. Martins Fontes (2000)

[3] Project, A.A.: - Autonomic Network Architectures. European Union IST 6th Framework Programme, http://www.ana-project.org (2011)

[4] AUTOI: Autonomic Internet Project. European Union IST 7th Framework Programme (2011)

[5] Baiôco, G., Costa, A., Calvi, C., Garcia, A.: IT Service Management and Governance - Modeling an ITSM Configuration Process: a Foundational Ontology Approach. Symposium on Integrated Network Management - SINM/IFIP/IEEE (2009)

[6] Barcelos, P.: et al.: OOTN - An Ontology Proposal for Optical Transport Networks. International Conference on Ultra Modern Telecommunications, IEEE Xplore, Print ISBN: 978-1-4244-3942-3 (2009)

[7] Caesar, M.: Identity-based routing. Technical Report No. UCB/EECS-2007-114 (2007)

[8] D'Ambrosio, M., Marchisio, M., Vercellone, V., Ahlgren, B., Dannewitz, C.: Second NetInf architecture description, http://www.4ward-project.eu (2010)

[9] Farinacci, D., Fuller, V., Meyer, D., Lewis, D.: LISP Project - Locator/ID Separation Protocol. Network Working Group, draft-ietf-lisp-06 (2010)

[10] FIND: Future Internet Design Program. National Science Foundation, http://www.nets-find.net (2011)

[11] FIRE: FIRE White Paper. Future Internet Research and Experimentation (2009)

[12] Ford, B.: UIA: A Global Connectivity Architecture for Mobile Personal Devices. Ph.D. Thesis. Massachusetts Institute of Technology, Dep. of Electrical Eng. and Comp. Sci (2008)

[13] Gasevic, D., Djuric, D., Devedzic, V.: Model Driven Engineering and Ontology Development, 2nd edn. Springer, Heidelberg (2009)

[14] GENI: Global Environment for Network Innovation Program. National Science Foundation, http://www.geni.net (2011)

[15] Gruber, T.: Toward Principles for the Design of Ontologies Used for Knowledge Sharing. International Journal of Human and Computer Studies, 43(5-6): 907928 (1995)

[16] ITU-T: Information Technology - Open Systems Interconnection - Application Layer Structure. Recommendation X.207 - ISO/IEC 9545:1993 (1993)

[17] Lacy, L.: OWL: Representing Information Using the Web Ontology Language. Trafford (2005)

[18] Lesniewski, S.: Comptes rendus des séances de la Société des Sciences et des Lettres de Varsovie. pp. 111-132 (1930)

[19] Malva, G.R., Dias, E.C., Oliveira, B.C., Pereira, J.H.S., Kofuji, S.T., Rosa, P.F.: Implementação do Protocolo FINLAN. In: 8th International Information and Telecommunication Technologies Symposium (2009) 
[20] Mealy, G.: Another look at data. In: Proceedings of the Fall Joint Computer Conference. AFIPS November 14-16, Volume 31, pp. 525-534. Thompson Books, Washington and Academic Press, London (1967)

[21] Pasquini, R., Paula, L., Verdi, F., Magalhães, M.: Domain Identifiers in a Next Generation Internet Architecture. In: IEEE Wireless Communications and Networking Conference - WCNC (2009)

[22] Pasquini, R., Verdi, F., Magalhães, M.: Towards a Landmark-based Flat Routing. In: 27th Brazilian Symposium on Computer Networks and Distributed Systems - SBRC (2009)

[23] Pereira, J.H.S., Kofuji, S.T., Rosa, P.F.: Distributed Systems Ontology. In: IEEE/IFIP New Technologies, Mobility and Security Conference (2009)

[24] Pereira, J.H.S., Kofuji, S.T., Rosa, P.F.: Horizontal Address Ontology in Internet Architecture. In: IEEE/IFIP New Technologies, Mobility and Security Conference (2009)

[25] Pereira, J., Sato, L., Rosa, P., Kofuji, S.: Network Headers Optimization for Distributed Programming. In: 9th International Information and Telecommunication Technologies Symposium (2010)

[26] Pereira, J.H.S., Kofuji, S.T., Rosa, P.F.: Horizontal Addressing by Title in a Next Generation Internet. In: IEEE International Conference on Networking and Services p. 7 (2010)

[27] Prudêncio, A., Willrich, R., Diaz, M., Tazi, S.: NetQoSOnt: Uma Ontologia para a Especificação Semântica de QoS em Redes de Computadores. In: 14o Workshop de Gerência e Operação de Redes e Serviços - WGRS-SBRC (2009)

[28] Rubio-Loyola, J., Serrat, J., Astorga, A., Chai, W.K., Galis, A., Clayman, S., Mamatas, L., Abid, M., Koumoutsos, G.: et al.: Autonomic Internet Framework Deliverable D6.3. Final Results of the AutonomicI Approach. AutoI Project (2010)

[29] Souza, J.: Lógica para Ciência da Computação. Campus (2008)

[30] Trossen, D., Nikander, P., Visala, K., Burbridge, T., Botham, P., Reason, C., Sarela, M., Lagutin, D., Koptchev, V.: Publish Subscribe Internet Routing Paradigm - PSIRP. Final Updated Architecture, Deliverable D2.5 (2010)

[31] Tselentis, G., et al.: Towards the Future Internet - A European Research Perspective. IOS Press, Amsterdam (2009)

[32] Tselentis, G., et al.: Towards the Future Internet - Emerging Trends from European Research. IOS Press, Amsterdam (2010)

[33] Tsiatsis, V., Gluhak, A., Bauge, T., Montagut, F., Bernat, J., Bauer, M., Villalonga, C., Barnaghi, P., Krco, S.: The SENSEI architecture-Enabling the Real World Internet. In: Towards the Future Internet, pp. 247-256. IOS Press, Amsterdam (2010)

[34] Vissers, C., Logrippo, L.: The Importance of the Service Concept in the Design of Data Communications Protocols. In: Proceedings of the IFIP WG6 1, 3 (1986)

[35] Wang, J., Guo, M., Camargo, J.: An Ontological Approach to Computer System Security. Information Security Journal: A Global Perspective (2010)

[36] Wong, W.: et al.: An Architecture for Mobility Support in a Next Generation Internet. In: The 22nd IEEE International Conference on Advanced Information, Networking and Applications - AINA (2008) 